\title{
Modification of fluorescent nanocrystals with 6-thioguanine: monitoring of drug delivery
}

\author{
Ilona Grabowska-Jadach ${ }^{1}$ (D) Marcin Drozd ${ }^{1,2} \cdot$ Dominika Kulpińska $^{1} \cdot$ Klaudia Komendacka $^{1} \cdot$ Mariusz Pietrzak $^{1}$
}

Received: 18 March 2019 / Accepted: 27 June 2019 / Published online: 11 July 2019

(c) The Author(s) 2019

\begin{abstract}
The purpose of the presented work was to modify the surface of core/shell-type $\mathrm{CdSe}_{x} \mathrm{~S}_{1-x} / \mathrm{ZnS}$ quantum dots (QDs) with an anticancer drug to form bifunctional nanoconjugate for imaging and drug delivery. 6-Thioguanine (6-TG) which is used to treat acute myeloid and lymphoblastic leukemia was applied for this aim. The modification of the nanocrystals was carried out using a biphasic method, based on transferring of QDs from the organic phase to the aqueous one with the simultaneous exchange of hydrophobic ligand to the hydrophilic one. To enable a complex evaluation of 6-TG bioactivity, the surface of quantum dots was also modified using mercaptoacetic acid (MAA). Mercaptoacetic acid was chosen as a biologically neutral ligand; so, it was possible to evaluate the effect of nanocrystals toxicity without the participation of the drug (6-TG ligand versus neutral ligand). Moreover, the effect of 6-TG itself on cells was also studied. The studies were carried out on three cell lines: K562 (human myeloid leukemia), A549 (human lung cancer cells) and MRC-5 (normal human lung cells). Evaluation of the cytotoxicity of nanostructures, at various concentrations, was carried out after 24- and 48-h incubation of the cell culture with nanoparticles/drug solutions. MTT and the Alamar Blue assays were used to determine cell viability. Based on the cytotoxicity measurements, it was found that quantum dots modified with 6-TG are more toxic than the drug itself or QDs modified with 3-mercaptoacetic acid. Images taken using fluorescence and confocal microscopy allowed to define the location of examined QDs inside cells.
\end{abstract}

Keywords Quantum dots · Cytotoxicity · 6-Thioguanine · Drug delivery · Drug distribution monitoring

\section{Introduction}

Quantum dots (QDs) are inorganic semiconductor nanocrystals, consisting of 100-1000 of atoms belonging typically to groups II and VI or III and V (e.g. InP, InAs) of the periodic table (e.g. CdSe, CdTe) (Zrazhevskiy et al. 2010). They are typically characterized with a diameter between 2 and $10 \mathrm{~nm}$, which is close to the exciton Bohr radius (electron-hole distance). This results in quantum confinement effects which influence the optical properties of QDs (as fluorophores) - the maximum of absorption and emission wavelength is shifted to the blue region as an effect of

Ilona Grabowska-Jadach

ilona@ch.pw.edu.pl

1 Chair of Medical Biotechnology, Faculty of Chemistry, Warsaw University of Technology, Noakowskiego 3 St., 00-664 Warsaw, Poland

2 Centre for Advanced Materials and Technologies CEZAMAT, Poleczki 19 St., 02-822 Warsaw, Poland reducing of QDs size (Bera et al. 2010; Bruchez 1998). The smaller is the diameter of QD (band gap energy increases), the light of higher energy is emitted (shorter wavelength). Thus, the size of the QDs is a key factor that determines the color of their fluorescence. Photoluminescence emission can occur in a wide spectrum of colors (from UV to the infrared region) and can be tuned by changing the QDs diameter. The wavelength of emitted fluorescence is also influenced by the chemical composition of both the core and the shell (if present). In general, QDs are characterized by: narrow emission peaks, wide absorption bands, high photoluminescence quantum yields and relatively large Stokes shift value (Gutiérrez-Lazos et al. 2012; Yeon Woo et al. 2012).

Taking into account the QDs structure, they can be divided into three groups: core, core/shell and core/multiple shell QDs. Shell used as coating material for the core has usually higher bandgap energy than the core material (Antonello et al. 2011; Gao 2011). The presence of this coating is significant because it improves the optical properties of the quantum dots, protects against degradation in 
the cellular environment (core passivation reduces nanoparticles sensitivity to environmental condition changes) and also reduces QDs toxicity (reduces metal ion leakage from the core). It should be noted that the surface of QDs can be modified with different types of ligands. The choice of appropriate ligand is a crucial parameter because the ligands affect stability, dispersibility and water solubility of QDs (Abdul Ghani et al. 2014). Despite the impact on the physical and chemical parameters, ligands can also influence QDs interaction with cells or living organisms (Biju et al. 2010; Jin and Hildebrandt 2012; Karabanovas et al. 2014). Therefore, the applied ligands often determine the applicability of these nanoparticles; for instance for biological or medical purposes, the ligands have to be carefully selected (Abbasi et al. 2015; Azzazy et al. 2007; Smith et al. 2008).

Therapeutic applications of nanoparticles (NPs) are very diverse. One of them is the treatment of cancer $(\mathrm{Li}$ and $\mathrm{Pu}$ 2019; Mottaghitalab et al. 2019; Xiao et al. 2019). Some chemotherapeutic drugs have already been successfully attached to the surface of nanoparticles. Examples of such drugs can be: doxorubicin, paclitaxel, etoposide and carboplatin (Nanjwade et al. 2010; Nehate et al. 2014; Singla et al. 2002; Xia et al. 2018). Due to the interesting properties of 6-TG, such as inhibiting purine synthesis, disrupting DNA synthesis and inducing cell apoptosis, we decided to use it for the modification of $\mathrm{CdSe}_{x} \mathrm{~S}_{1-x} / \mathrm{ZnS}$ surface. Since 1988, when G.H. Hitchings, J.W. Black and G.B. Elion received the Nobel Prize in physiology and medicine for formulating significant principles of pharmacological treatment of tumor (especially guidelines for chemotherapy) and creating a new generation of anticancer drugs (among others 6-TG), 6-TG remains the object of a of great interest. This compound belongs to a thionucleobases group which are an important class of FDA-approved, antimetabolite-type anti-cancer drugs, but their administration is not free of side effects. Sulfur atom present in thiopurines' structure exhibits high affinity towards sulfhydryl moieties of biogenic thiols and plasma proteins, which reduces the effectiveness of therapy and biological lifetime (Aghevlian et al. 2013). It was also proven that long-term treatment with purine analogues in combination with sun exposure leads to substantial (50-200fold) increase of skin cancer morbidity (Wang et al. 2016). The issue of UVA-induced oxidation side reaction of thiopurines to mutagenic and cytotoxic guanine-6-sulfonate is one of the most important limitations of 6-TG implementation in biological systems (Zou et al. 2013). To overcome dose- and phototoxicity-dependent side effects associated with the use of thiopurine drug family, several approaches were developed, including administration in the form of metal-drug complexes (Chalmers et al. 1972) and nanoparticle-based platforms for enhanced delivery for chemotherapies (Sleightholm et al. 2011; Wang et al. 2016). The conjugation of 6-TG to nanocarrier can help to partially eliminate several limitations affecting low concentration of bioactive form, such as poor availability after oral administration, fast renal clearance after intraveneous administration and severe side effects e.g. myelosuppression. It was reported that the protection of sulfur-containing moiety improves pharmacokinetics of 6-TG, modifies its photochemistry and thus substantially diminishes photosensitization ability of this compound (Zhang et al. 2011). Nanoparticles are considered as an attractive carrier due to their size-dependent, preferential accumulation in tumor sites caused by EPR effect related with increased permeability of tumor tissues. Such factors improve total antiproliferative activity of 6-TG drug. Wang et al. described the methodology of photophysical and photochemical properties modification of 6-TG by its loading onto gold nanoparticles which resulted in the impairment of the 6-TG-triggered DNA oxidative damage (Wang et al. 2016).

In the current work, there were obtained two kinds of QDs $\left(\mathrm{CdSe}_{x} \mathrm{~S}_{1-x} / \mathrm{ZnS}-6-\mathrm{TG}\right.$ and $\left.\mathrm{CdSe}_{x} \mathrm{~S}_{1-x} / \mathrm{ZnS}-\mathrm{MAA}\right)$ and this way, we were able to check the impact of the drug bound to the nanoparticles on human cells (in vitro studies). To quantitatively evaluate the influence of attachment of chemotherapeutic to QDs surface on its cytotoxicity, biological activity of such modified QDs and 6-TG solutions was examined. The modification of nanocrystals was conducted using the interphasic transfer method, based on a migration of QDs from organic to aqueous phase together with simultaneous replacement with of hydrophobic ligands to hydrophilic 6-TG. The optical properties of prepared nanocrystals were examined using spectrophotometry and spectrofluorimetry. The tests of viability were performed on three selected human cell lines: K562 (myeloid leukemia), A549 (lung cancer cells) and MRC-5 (normal lung cells). Moreover, the morphology of cells and QDs distribution based on the microscope images after the incubation with modified QDs was examined.

\section{Materials and methods}

\section{Materials}

$\mathrm{CdSe}_{x} \mathrm{~S}_{1-x}$ mixed-core-type nanocrystals coated with $\mathrm{ZnS}$ and stabilized with oleic acid (6-nm diameter, solution in toluene, $\lambda_{\mathrm{em}}=630 \mathrm{~nm}$ ) were purchased from Cytodiagnostics (Burlington, Canada). 6-thioguanine and mercaptoacetic acid were purchased from Sigma Merck (Poznań, Poland). Other chemicals used throughout QDs modification were all purchased from Sigma to Merck or Thermo Fisher GmbHformerly Alfa Aesar (Karlsruhe, Germany) and used as received. Milli-Q water (resistivity $\geq 18 \mathrm{M} \Omega \mathrm{cm}$ ) was used throughout preparation of all solutions and buffers unless stated otherwise. 
Phosphate-buffered saline (PBS), Minimum Essential Medium Eagle (MEME), antibiotics (penicillin and streptomycin), calcein AM, MTT reagent, MEM Non Essential Amino Acids Solution, RPMI 1640 medium, L-glutamine were purchased from Sigma Aldrich. Medium MEME without phenol red, Tryple Express, fetal bovine serum (FBS) and trypan blue were purchased from Life Technologies. Chloroform, ethyl alcohol $96 \%$ solution, sodium hydroxide were purchased from POCh. We also used ethanol $70 \%$ (for sterilization) from Linegal Chemicals, mercury(II) sulphate from Merck and dimethyl sulfoxide (DMSO) from ChemPur. BBS buffer $\mathrm{pH} 9.0$ and $0.2 \mathrm{M}$ acetate buffer $\mathrm{pH} 4.6$ were also used in QDs studies.

\section{Cell lines}

Human cell lines were used in our studies: A549 (adenocarcinomic alveolar basal epithelial cells), MRC-5 (fibroblasts derived from lung) and K562 (chronic myelogenous leukemia). The adherent MRC-5 and A549 cell lines were collected from European Collection Cell Cultures, K562 suspension cells were purchased from Sigma Aldrich.

\section{Cell culture}

The A549 cells were grown in Minimum Essential Medium Eagle supplemented with $10 \%$ of fetal bovine serum, $1 \%$ volume of 25-mM L-glutamine, $1 \%$ volume of 100-mM penicillin and streptomycin. The same culture medium containing $0.01 \%$ amino acid solution was used for MRC- 5 cells. The K562 cells were grown in RPMI 1640 medium containing $10 \%$ volume of fetal bovine serum, $1 \%$ volume of $25-\mathrm{mM}$ L-glutamine, $1 \%$ volume of $100-\mathrm{mM}$ penicillin and streptomycin. Cell cultures were incubated at $37{ }^{\circ} \mathrm{C}$ and $5 \% \mathrm{CO}_{2}$ in a humidified atmosphere. Cells were sub-cultured when cell culture reached $90-100 \%$ of confluence (it was about three times a week).

\section{Modification of QDs with 6-TG and MAA ligands and QDs purification}

The hydrophilization of QDs surface was accomplished by means of ligand exchange procedure in biphasic system with the use of 6-TG as a capping agent. Briefly, $2 \mathrm{~mL}$ of QDs suspension in toluene was fourfold diluted with absolute ethanol, followed by centrifugation $(15 \mathrm{~min} \times 15,000 \mathrm{rpm})$ to remove primary solvent. Then, the obtained pellet was resuspended in $2 \mathrm{~mL}$ of chloroform. 10-mM aqueous solution of TG was prepared by addition of respective amount of solid and further alkalization of the mixture with 1-M tetramethylammonium hydroxide (TMAH), which is required to enhance 6-TG solubility. The $\mathrm{pH}$ of this solution was adjusted to 11.4 with TMAH. $2 \mathrm{~mL}$ of as obtained aqueous
6-TG solution in glassy vial was purged with a stream of nitrogen and then combined with an aliquot of chloroformic QDs solution in the absence of air. The biphasic system was vigorously shaken at room temperature and in the absence of light until bottom phase (chloroform) became colorless (average time-12 h). Water-soluble QDs (upper phase) were subjected to purification by dialysis against $1.5 \mathrm{~L}$ of $1-\mathrm{M}$ borate buffer containing $15 \mathrm{mM} \mathrm{NaCl}(\mathrm{pH} 9.0)$. The dialysis was performed in tubing made of benzoylated cellulose (MWCO $=2 \mathrm{kDa}$, Sigma-Merck) for $3 \mathrm{~h}$. To remove fraction of aggregates and particulate matter, dialyzed QDs were centrifuged $(10 \mathrm{~min} \times 5000 \mathrm{rpm})$ and the reddish supernatant was filtered using a syringe filter made of regenerated cellulose, pore size $=0.22 \mu \mathrm{m}$ (Filtrakon, Poland) . As obtained, 6-TG-coated quantum dots (QD-6-TG) were stored in $4{ }^{\circ} \mathrm{C}$ in the darkness and used for biological studies. No signs of precipitation were observed for at least 4 weeks of storage. To obtain MAA-capped $\mathrm{CdSe}_{x} \mathrm{~S}_{1-x} / \mathrm{ZnS}$ QDs, the procedure described in our previous report was exploited (Kalinowska et al. 2018).

\section{Concentration of quantum dots and 6-TG-analysis of $\mathrm{Cd}^{2+}$ and 6-TG content in QD-6-TG samples}

To quantitatively evaluate the contribution of both nanocrystals and cytotoxic drug used as nanocrystal capping ligand to overall cytotoxic effect, the concentration of two most toxic agents (cadmium ion and 6-TG) in QDs sample was determined. To release $\mathrm{Cd}^{2+}$ and free 6-TG from nanoconjugates, QDs were dissolved in $2-\mathrm{M}$ hydrochloric acid $(100 \mu \mathrm{L}$ of QDs $+900 \mu \mathrm{L}$ of acid). The obtained mixture was sonicated for $20 \mathrm{~min}$ to finalize acidic digestion of nanocrystals. Cadmium ion was determined by means of anodic stripping voltammetry according to methodology described in our previous reports (Kalinowska et al. 2018). The method of multiple standard addition (min. four addition per each measurement) was applied. To calculate average cadmium content in QDs sample, at least four independent replications were made. DPSV measurements were carried out with the use of CHI 1040A electrochemical workstation (USA). Three-electrode system consisting of glassy carbon disk electrode (as mercury film electrode substrate), $\mathrm{Ag} / \mathrm{AgCl} / 3 \mathrm{M} \mathrm{KCl}$ (reference electrode) and gold wire (auxiliary electrode) was applied. 0.2-M acetate buffer $\mathrm{pH} 4.6$ containing $100-\mu \mathrm{M} \mathrm{HgSO}_{4}$ was used as a supporting electrolyte. Supporting electrolyte was purged with nitrogen prior to use and blanketed with nitrogen stream during electrochemical measurements to avoid reabsorption of oxygen. The applied parameters were as follows: cadmium deposition potential $-0.9 \mathrm{~V}$, cadmium deposition time $=1 \mathrm{~min}$, stripping potential range -0.75 to

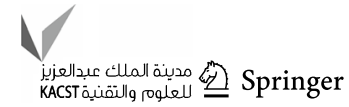


$-0.4 \mathrm{~V}$, DPSV amplitude $=25 \mathrm{mV}$, pulse time $=0.02 \mathrm{~s}$, increment $0.04 \mathrm{~V}$, period $=0.04 \mathrm{~s}$, quiet time $=10 \mathrm{~s}$. The exemplary voltammograms and standard addition curve are depicted in Fig. 1a.

Free 6-TG was determined spectrophotometrically $\left(\lambda_{\max } 6-\mathrm{TG}=348 \mathrm{~nm}\right)$ after appropriate dilution of aciddissolved QDs sample with 2-M HCl. A set of 6-TG solutions of various concentrations in 2-M hydrochloric acid was used for the calibration curve preparation (see Fig. 1b). The determined concentrations of cadmium ion and 6-TG were required to prepare appropriate dilutions of QD-6-TG and 6-TG administered to cell lines.

\section{Physicochemical properties of QDs after surface modification}

Registration of UV-Vis absorption spectra was accomplished using Lambda25 Perkin-Elmer spectrophotometer (USA) and quartz cuvettes of path length $=1 \mathrm{~cm}$. Photoluminescence absorption spectra were captured with fluorescence spectrophotometer Fluoromax 3 Horiba Jobin-Yvon (France) at excitation wavelength of $488 \mathrm{~nm}$ (slit widths for emission and excitation $=2 \mathrm{~nm}$ ) in quartz cuvettes (path length $=1 \mathrm{~cm}$ ). Tracking the changes in hydrodynamic diameters (before and after ligand exchange) as well as zeta-potential measurements were carried out using Malvern Zetasizer Nano ZS instrument (UK), equipped with $\mathrm{He}-\mathrm{Ne}$ laser (wavelength $=632.8 \mathrm{~nm}$, power $=5 \mathrm{~mW}$ ) and fluorescence attenuator. DLS measurements were conducted in quartz microcuvettes of volume $50 \mu \mathrm{L}$ (Hellma). Zeta potential was determined using universal dip cell in disposable PS cuvettes.

(a)

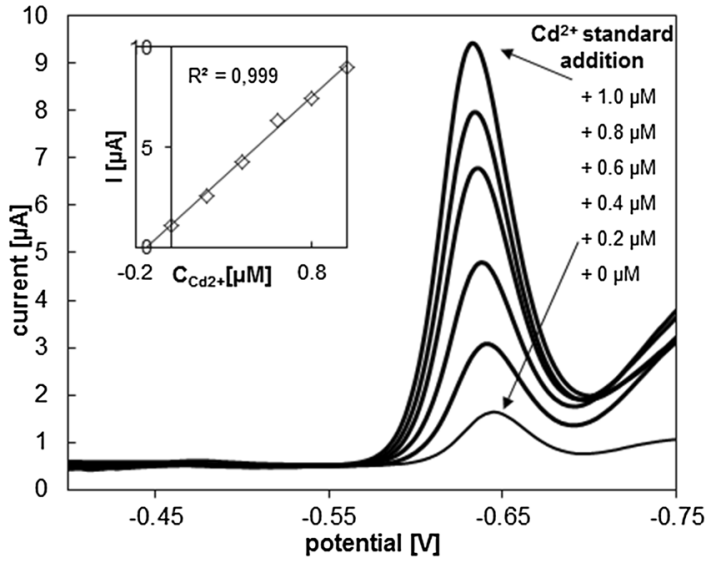

Fig. 1 a Exemplary DPSV voltammograms representing the consecutive additions of $\mathrm{Cd}^{2+}$ during determination of released cadmium ions after QDs acidic dissolution. Inset shows the corresponding standard addition curve. The mean of three independent measurements

\section{QDs cytotoxicity evaluation using MTT test}

MTT is a colorimetric test used for in vitro evaluation of the viability of cells. We used this test for analysis of adherent cells (MRC-5 and A549) after incubation of the cell culture with quantum dots/drug solutions. This test is based on a reduction of tetrazolium salt (MTT, 3-(4,5-dimethylthiazol-2-yl)-2,5-diphenyltetrazolium bromide) to formazan by dehydrogenases, which are present in the mitochondria of living cells. The amount of the formed colored formazan is proportional to the number of living cells and is typically determined by absorbance measurements. The cytotoxicity test takes 3-4 days, depending on the time of incubation with nanoparticles ( $24 \mathrm{~h}$ or $48 \mathrm{~h}$ ). At the beginning, cell suspensions of appropriate density $\left(1.5 \times 10^{5}\right.$ for A549 and $2 \times 10^{5}$ cells/mL for MRC-5) were prepared and $100 \mu \mathrm{l}$ of such suspension was placed to each well of 96-well plate. After $24 \mathrm{~h}$ (cell attachment to surface of the well plate was observed) medium was removed and $100 \mu$ of solutions of QD-6-TG/6-TG (prepared in MEME culture medium without phenol red) were placed to each well. The applied concentrations of modified quantum dots (QD-6-TG and QDMAA) were as follows: 5, 25, 50, 75, $100 \mu \mathrm{M}$. Moreover, the response of the cells to the increasing concentrations of 6-TG: $15,75,150,225,300 \mu \mathrm{M}$ was checked in our tests. The used 6-TG concentrations corresponded to the amount of drug attached to the surface of the nanoparticles. The control sample for each cell line-cell culture with medium without QDs or drug was also prepared. Incubation at $37^{\circ} \mathrm{C}$, in a humidified atmosphere $\left(5 \% \mathrm{CO}_{2}\right)$, was carried out for $24 \mathrm{~h}$ and $48 \mathrm{~h}$. After the incubation, solutions were removed using a syringe with a blunt-ended needle. To six wells containing cell cultures, $100 \mu \mathrm{L}$ of the 8 -nM calcein AM

(b)

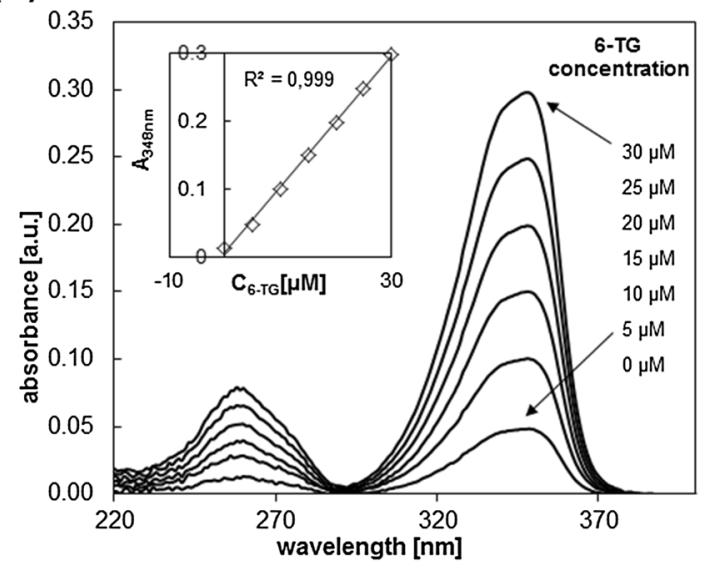

was used as valid stock concentration for further QDs dilutions. b UV absorption spectra of 6-TG in different concentrations (from 0 to $30 \mu \mathrm{M}$ ) in $2 \mathrm{M} \mathrm{HCl}$. Inset shows the calibration curve based on the absorbance intensities at $348 \mathrm{~nm}$ 
solution $\left(\lambda_{\mathrm{ex}}=490 \mathrm{~nm}, \lambda_{\mathrm{em}}=515 \mathrm{~nm}\right)$ in PBS was added. $100 \mu \mathrm{L}$ of MTT solution in PBS $(0.5 \mathrm{mg} / \mathrm{mL})$ was added into the remaining wells. The cells with calcein-AM dye solution were incubated for $20 \mathrm{~min}$ in an incubator. Then, the medium was removed and $100 \mu \mathrm{L}$ of PBS was added to each well. Cell images were captured using Olympus inverted fluorescence microscope. Cells with the MTT solution were incubated for $4 \mathrm{~h}$ in the incubator (protected from light). After incubation, MTT solution was removed and $100 \mu \mathrm{L}$ of DMSO was added to each well due to dissolve the formazan crystals that were formed. The final step in MTT test was the measurement of the absorbance at $570 \mathrm{~nm}$ using multimode reader Cytation 3 (BioTek). The results were presented as the percentage of cells viability compared to the control sample (cell culture incubated with medium):

Cells viability $=A_{\mathrm{S}} / A_{\mathrm{C}} \times 100 \%$,

where $A_{\mathrm{S}}$ is the average absorbance of samples, $A_{\mathrm{C}}$ is the average absorbance of control sample.

\section{Cytotoxicity studies using Alamar Blue test}

The viability of suspension cells (K562) was evaluated using Alamar Blue test. In this resazurin-based test fluorescence of formed resorufin is measured. Living cells possess ability to reduce the blue and non-fluorescent resazurin to red and highly fluorescent resorufin. The fluorescence signal is proportional to the number of living cells.

K562 line cells were seeded at a density of $5 \times 10^{5}$ cells/ $\mathrm{mL}$ in a volume of $100 \mu \mathrm{L}$ per well. Solutions of surfacemodified quantum dots or 6-TG drug were added to each well after seeding the cells. After adding the solutions to the cells, the concentrations of QDs in wells were as follows: $5 \mu \mathrm{M}, 25 \mu \mathrm{M}, 50 \mu \mathrm{M}, 75 \mu \mathrm{M}$ and $100 \mu \mathrm{M}(15,75,150,225$, $300 \mu \mathrm{M})$. Then the incubation of cell culture with tested nanoparticles/compounds was carried out. Finally, $5 \mu \mathrm{L}$ of Alamar Blue reagent (2.5\% final concentration) was added to each well and was incubated with cells for $4 \mathrm{~h}$. Then, the fluorescence intensity $\left(\lambda_{\mathrm{ex}}=552 \mathrm{~nm}, \lambda_{\mathrm{em}}=582 \mathrm{~nm}\right)$ was measured.

\section{Monitoring of a QD-6-TG distribution}

The equipment used for cell culture observation was as follows: Olympus CKX41 optical microscope, Olympus IX71 fluorescence microscope, and BioTek microplate reader. The confocal microscope was used to monitor QD-6-TG distribution within a single cell after 24-h and 48-h incubation of cell culture with the nanoparticles. For this purpose, cells were seeded on a four-sided (glass bottom) Petri dish. Samples of $500 \mu \mathrm{L}$ or $250 \mu \mathrm{L}$ of cell suspension (adherent cells or K562 cells, respectively) at densities described above were added to each well of dish. Then, QDs solution
( $250 \mu \mathrm{L}$ ) of chosen concentration was added to cell culture. In the case of adherent cell lines, before addition of QDs solution, the cells were incubated for $24 \mathrm{~h}$ in an incubator to allow them to adhere to the Petri dish surface. Then the medium was removed and QDs solution $(500 \mu \mathrm{L})$ of chosen concentration was added to the cell culture.

\section{Results and discussion}

Herein, we report the use of the anticancer and antileukemic drug-6-TG for the modification of fluorescent semiconductor nanocrystals (this drug has been used for such aim for the first time). To date, the only described approach of modification of nanomaterial with 6-TG (as well as other thiopurine analogues) was the modification of gold nanoparticles (Podsiadlo et al. 2007; Wang et al. 2016). The applied procedure of ligand exchange using thiolated derivative of guanine was successful, which was manifested by the migration of quantum dots from the bottom organic phase to the aqueous solution (see Fig. 2). The affinity of 6-TG ligand to QDs surface can be mainly attributed to the interaction of sulfur (C6) atom with $\mathrm{ZnS}$ shell. It should be emphasized, that in opposition to noble metal surfaces, the contribution of electron donor atoms (heterocyclic and acyclic nitrogens) is relatively small (Li et al. 2016; Podsiadlo et al. 2007). The important factor facilitating the establishment of bond between $\mathrm{ZnS}$ and the ligand is the alkalization of the exchange mixture, and thus deprotonation of 6-TG ligand. The higher binding energy between $\mathrm{ZnS}$ and the thiolate anion in comparison to -SH moiety was already reported for other bifunctional thiols (Tamang et al. 2011). It should be noted, that 6-TG,

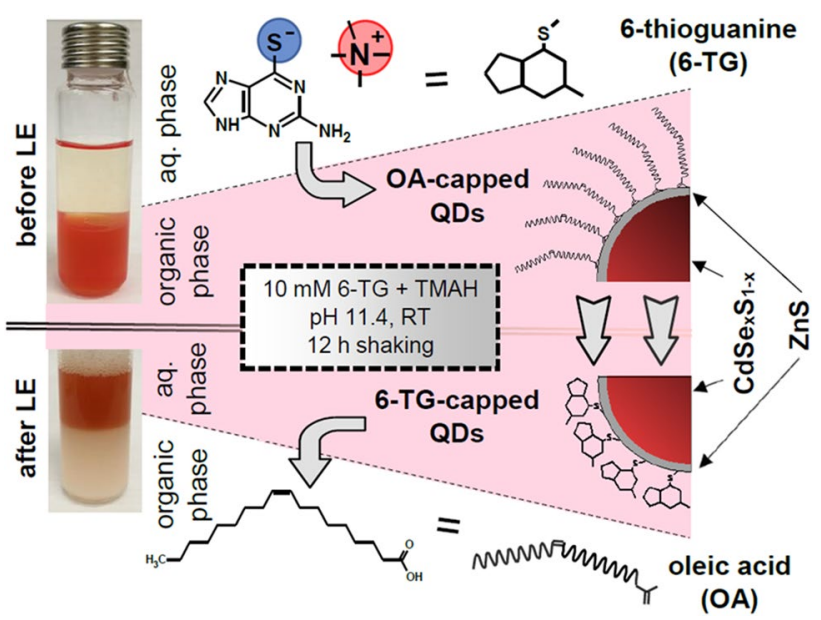

Fig. 2 Scheme of mechanism and procedure for $\mathrm{CdSe}_{x} \mathrm{~S}_{1-x} / \mathrm{ZnS}$ quantum dots ligand exchange. Picture shows images of biphasic system before and after ligand exchange (left), general structures of nanocrystal substrate (oleic acid-capped) and obtained QD-6-TG nanoconjugate (right)

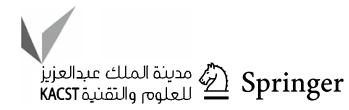


despite existing typically in a thione form, after deprotonation in alkaline media undergoes rapid tautomerization to thiolate (Fig. 2) (Li et al. 2016).

Both QDs solutions after ligand exchange gained excellent hydrophilicity and long-term stability in aqueous solutions, which make them suitable for the application in biological studies. The dispersion and photophysical properties of QDs after modification were examined by means of UV-Vis spectrophotometry, spectrofluorimetry and dynamic light scattering. The absorption (a) and emission spectra (b) of obtained QD-6-TG and QD-MAA are depicted in Fig. 3. In both cases, the lucid QD suspensions exhibiting apparent, sharp excitonic peaks were obtained. According to the analysis of PL spectra, the attachment of 6-TG leads to higher efficiency of emission for the same concentration of nanocrystals in comparison to short carboxylic ligand-MAA.

The calculated photoluminescence quantum yield of QD-6-TG [based on rhodamine 6G as reference dye (Deka et al. 2009)] amounted to $8.34 \%$. The modification also substantially affected the hydrodynamic diameters of obtained nanocrystals. According to DLS measurements, the mean diameter of MAA-capped QDs was $9.5 \mathrm{~nm}$; whereas, the diameter of QD-6-TG was substantially higher and amounted to $17.4 \mathrm{~nm}$. The substantial increase of hydrodynamic diameter in the case of 6-TG-modified nanocrystals can be explained by different thickness of ligand layers. Additionally, it should be underlined, that due to the chemical character of 6-TG (occurrence of aromatic rings), the additional effect of $\pi-\pi$-stacking may contribute to formation of multilayers. Such phenomenon has been already reported by Sleightholm et al. for 6-TG functionalized gold nanoparticles (Sleightholm et al. 2011). Both types of nanocrystals were characterized by anionic surface charge. Conducted measurements revealed that the surface zeta potential was
$-63.8 \pm 1.5 \mathrm{mV}$ for 6-TG-capped QDs and $-40.4 \pm 2.1 \mathrm{mV}$ for MAA-capped ones.

The cytotoxicity of nanoparticles modified with 6-TG on selected cell lines: A549, MRC-5 and K562 was evaluated using MTT or Alamar Blue tests. In this way, the cytotoxicity of $\mathrm{CdSe}_{x} \mathrm{~S}_{1-x} / \mathrm{ZnS}-6-\mathrm{TG}$ to normal and cancer cells was studied. The incubation of cells with tested quantum dots or 6-TG was carried out for 24 and $48 \mathrm{~h}$. The obtained results of observed cytotoxicity are presented in Fig. 4. The control sample represents $100 \%$ of viability. In accordance to ISO 10993-1:2018 norm (ISO 1099), a non-toxic concentration was ascertained for all tests, which were distinguished with more than $70 \%$ of cells viability.

The determined molar ratio of 6-TG to $\mathrm{Cd}^{2+}$ was close to 3.0:1.0. Therefore, to better illustrate the influence of particular components of modified QDs, respective concentration of 6-TG (three times higher than $\mathrm{C}_{\mathrm{Cd}}^{2+}$ ) was administered to cells during cytotoxicity studies.

In the case of A549 cell line (Fig. 4a, b), for both incubation times, the only non-toxic $\mathrm{CdSe}_{x} \mathrm{~S}_{1-x} / \mathrm{ZnS}-6-\mathrm{TG}$ concentration was $5 \mu \mathrm{M}$. For these nanoparticles, at such concentration, the cells viability amounted to $78.2 \%$ (24 h) and $77.4 \%$ (48 h). For $\mathrm{CdSe}_{x} \mathrm{~S}_{1-x} / \mathrm{ZnS}-\mathrm{MAA}$, the highest non-toxic concentration after 24 -h and 48-h incubation was $25 \mu \mathrm{M}(79.5 \%$ and $70.3 \%)$, while 6-TG showed the non-toxic effect in both cases at $15-\mu \mathrm{M}$ concentration (81.8 and $71.3 \%$, respectively). For MRC-5 cell line for the incubation lasting $24 \mathrm{~h}$ and $48 \mathrm{~h}$, the highest non-toxic $\mathrm{CdSe}_{x} \mathrm{~S}_{1-x} / \mathrm{ZnS}-6-\mathrm{TG}$ concentration was found to be $25 \mu \mathrm{M}(74.8 \%$ and $71.7 \%$, respectively) (Fig. 4c, d). The highest non-toxic concentration of $\mathrm{CdSe}_{x} \mathrm{~S}_{1-x} / \mathrm{ZnS}-\mathrm{MAA}$ as well as the aqueous solution of 6-TG after 24-h incubation was $50 \mu \mathrm{M}(70.4 \%)$ and $150 \mu \mathrm{M}(72.1 \%)$, respectively. However, for the extended incubation time (two times longer), the values of non-toxic (a)

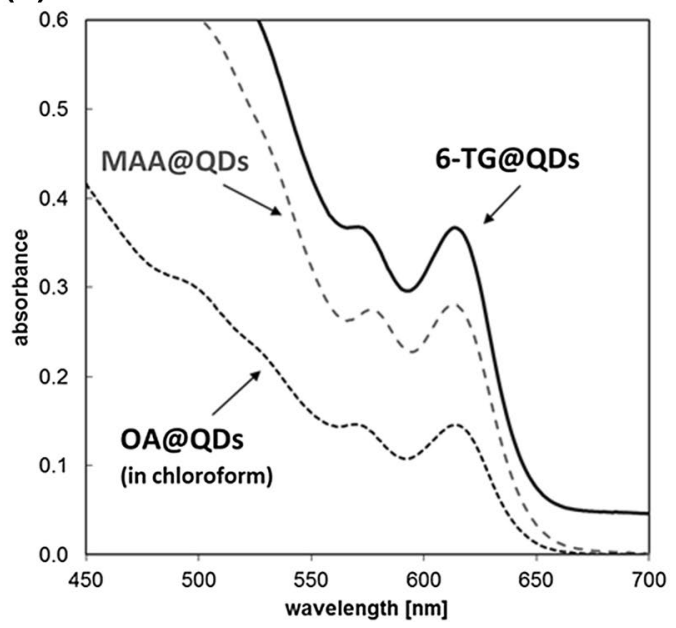

(b)

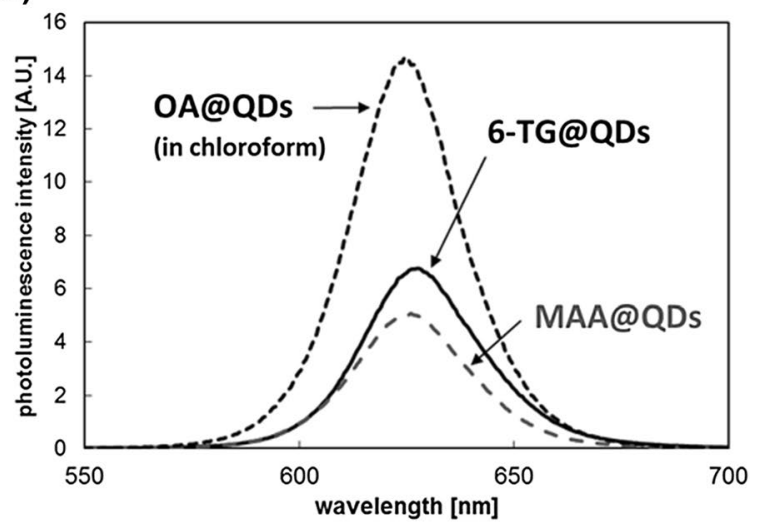

Fig. 3 UV-Vis absorption (a) and fluorescence emission spectra $\left(\lambda_{\mathrm{ex}}=488 \mathrm{~nm}\right)$ of modified QDs in aqueous solution $\left(C_{\mathrm{Cd}}^{2+}=100 \mu \mathrm{M}\right)$

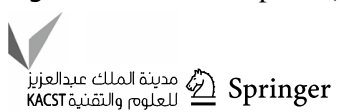


(a)

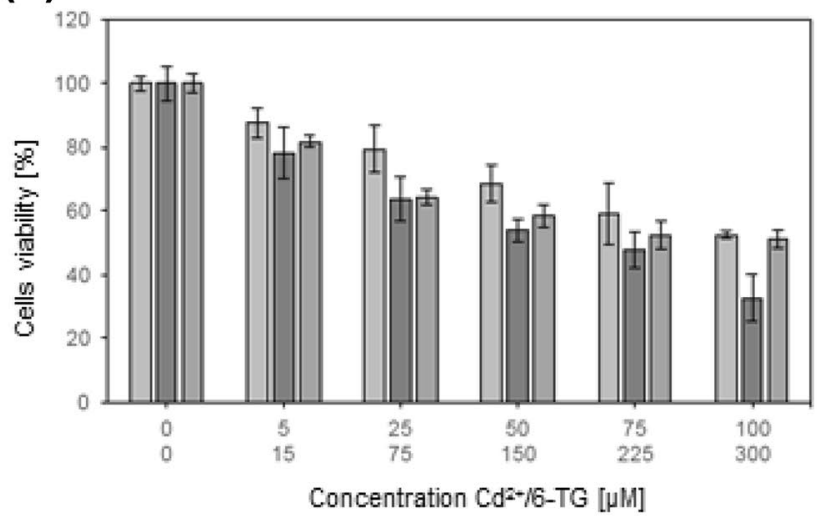

(c)

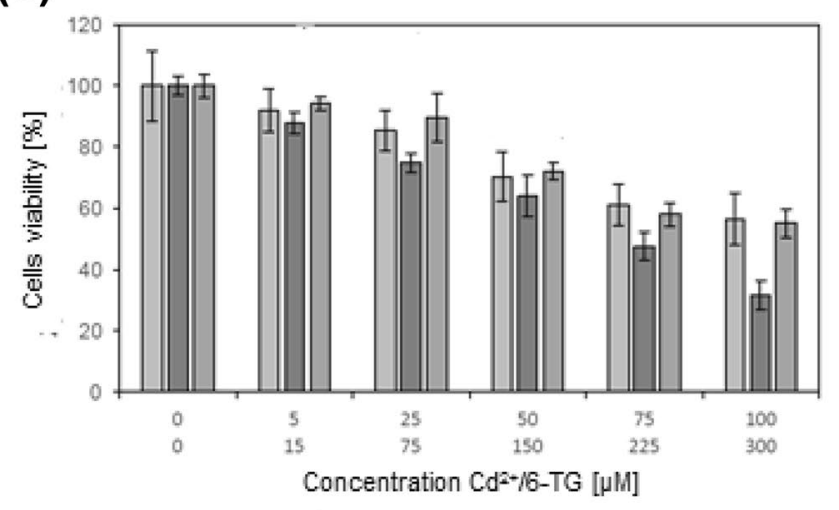

(e)

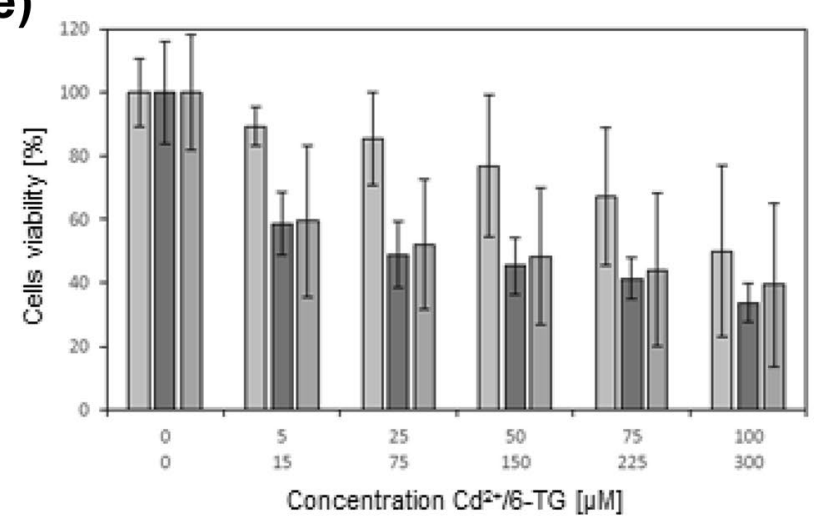

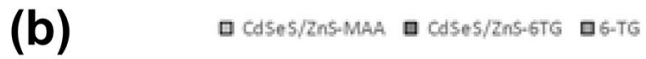

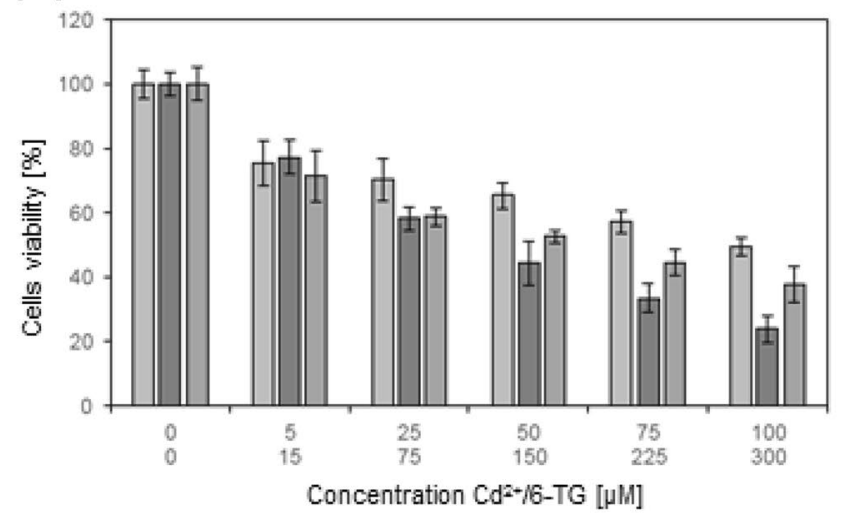

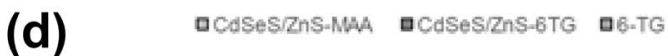

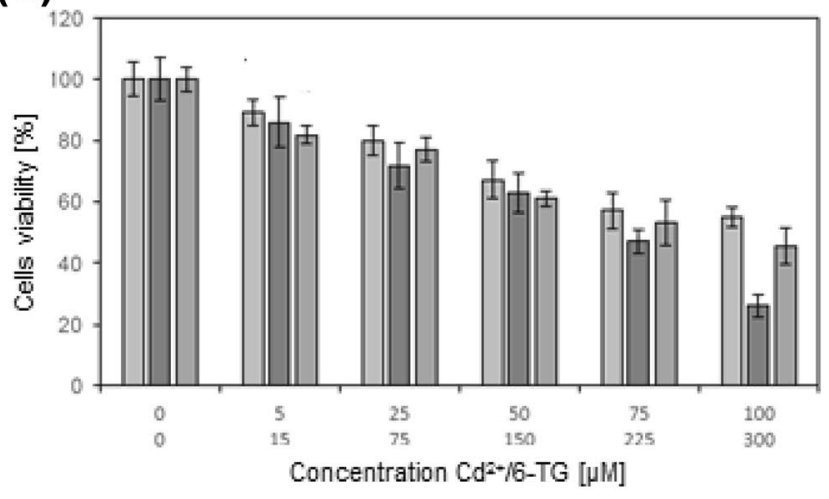

(f)

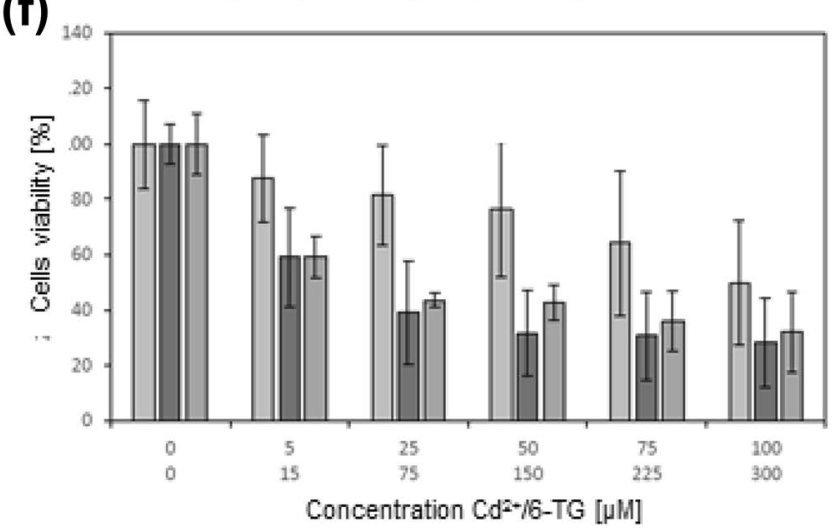

Fig. 4 Cells viability after incubation with QD-6-TG: a A549 cells (24 h), b A549 cells (48 h), c MRC-5 cells (24 h), d MRC-5 cells (48 h), e K562 cells (24 h), f K562 cells ( 48 h)

concentrations decreased twice and it was $25 \mu \mathrm{M}(80 \%)$ and $75 \mu \mathrm{M}(77.1 \%)$, respectively. Results of K562 exposition to the drug and nanoparticles solution are presented in Fig. 4e, f. Based on the observed low viability of K562 cells, it was concluded that $\mathrm{CdSe}_{x} \mathrm{~S}_{1-x} / \mathrm{ZnS}-6-\mathrm{TG}$ and the drug itself were toxic in the whole range of examined concentrations for these cells; whereas, the MAA-modified QDs were found to be non-toxic at concentrations of $50 \mu \mathrm{M}(76 \%)$ and lower. MAA is a biologically neutral ligand, so it can be concluded that cytotoxicity of these nanoparticles derived only from QDs composition. In our opinion, the release of toxic cadmium ions from the core of the quantum dot is the main factor affecting the toxicity of $\mathrm{CdSe}_{x} \mathrm{~S}_{1-x} / \mathrm{ZnS}$ QDs.

The fact that drug-modified quantum dots are more toxic than the drug itself suggests the contributing effect of the release of toxic cations from the core of quantum dots. Enhanced cytotoxicity may also be caused by more efficient drug transport into cells-nanoparticles act as drug delivery 
platforms. Similar observations were obtained by Selveraj et al. in studies on the cytotoxicity of 6-TG-modified gold nanoparticles on human epithelium Hep-2 cells (Selvaraj et al. 2010). The Au-6-TG complex induced higher cytotoxicity than the same amount of the drug itself.

We compared the biological activity of QDs with surface modified with 6-TG on three different cell lines and the lowest decrease in cells viability was observed for MRC-5 cells (Fig. 5). The lowest viability was found for human leukemia cells (K562), which is in line with our expectations, because the applied ligand (6-TG) is a cytostatic drug (an antimetabolite) belonging to the purine analogs. This drug is used for acute leukemias (especially acute myeloid leukemia and acute lymphoblastic leukemia) treatment. Thioguanine is incorporated into DNA and RNA, which contributes to its cytotoxic action.

In the next stage of the research, we checked how nanoparticles affect the morphology of cells. We conducted these studies for both adherent cell lines. In Fig. 6, selected microscopic images are presented. On their basis, it was found out that the increased concentration of $\mathrm{CdSe}_{x} \mathrm{~S}_{1-x} / \mathrm{ZnS}-6-\mathrm{TG}$ as well as longer incubation time changes the morphology of cells and inhibits their growth. The shape of A549 cells in the logarithmic growth phase is similar to a triangle. In images of A549 cell culture incubated with solutions of QD-6-TG, a change in cell morphology is noticeable. (a)

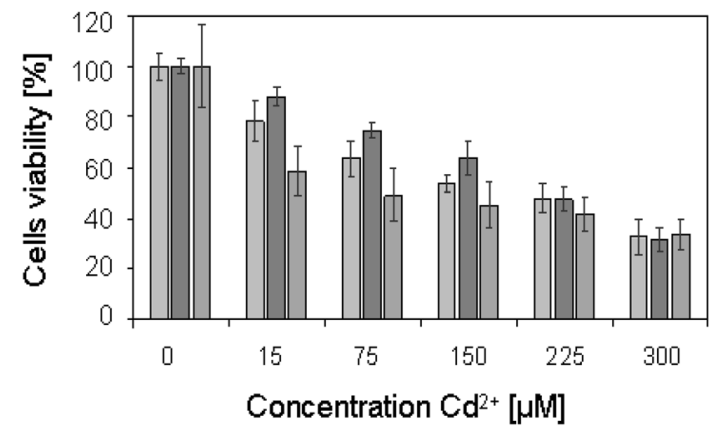

(b)

ㅁ. A549 $\square$ MRC5 $\square \mathrm{K} 562$

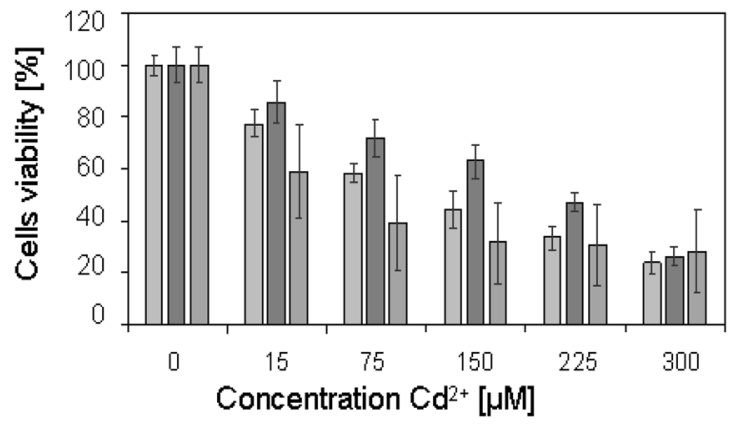

Fig. 5 Cells viability after: $\mathbf{a} 24 \mathrm{~h}$ of incubation and $\mathbf{b} 48 \mathrm{~h}$ of incubation with QD-6-TG

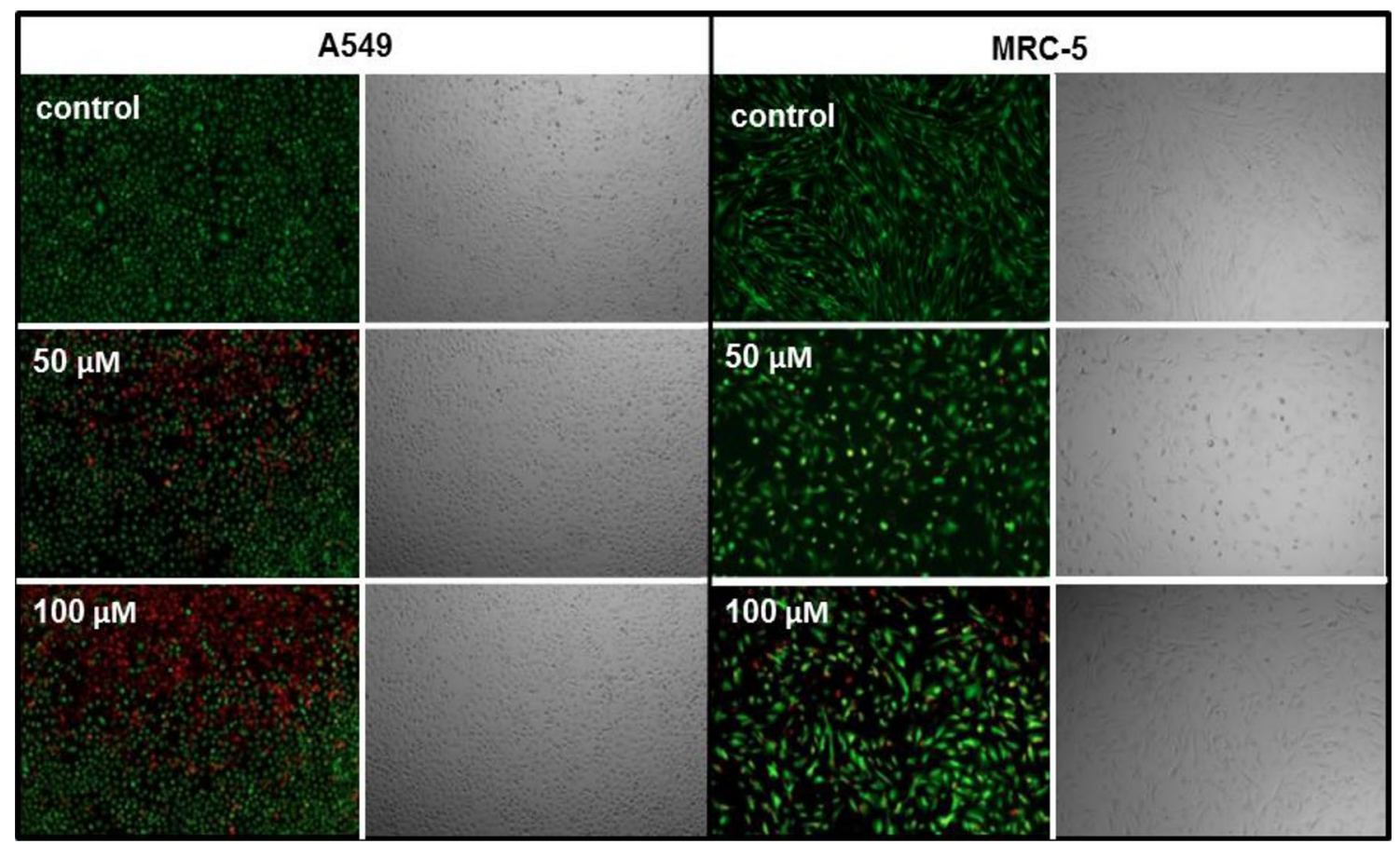

Fig. 6 A549 and MRC-5cells morphology after 24-h incubation with QD-6-TG 
The edges of the cells are irregular or cells became oval. In contrast, MRC-5 cells in the logarithmic growth phase are elongated and narrowed at the ends. At high concentrations of QD-6-TG, these cells lose the spindle shape, in favor to irregular, bulging structure. The cellular shrinkage was also observed, which may indicate, that the cell death proceeds through apoptosis. When in contact with solutions of higher concentrations of QDs, both types of cells exhibited deteriorated adhesion to the substrate. The lower density of cells is also visible as the effect of increased concentration of QDs. When the incubation lasted $48 \mathrm{~h}$, the changes described above were more visible.

To track the distribution of nanoparticles inside cells, confocal microscope images were taken (Fig. 7). The cells nuclei have been stained with Hoechst 33342. It was observed that for normal and cancer lung cells, penetration of quantum dots into the cells occurred. However, for both tested incubation times ( $24 \mathrm{~h}$ and $48 \mathrm{~h}$ ), no penetration of nanoparticles into the cell nucleus was observed. In addition, nuclei shrinkage was observed.

The pictures show the localization of quantum dots (observed as fluorescence emission) around and inside cells, which is a proof that QDs were attached onto cell membrane or penetrated into the cells. Our observations are consistent with those described in the work on interactions of fluorescent crystalline $\mathrm{ZnO}$ hexagonal nanosheets with $\mathrm{K} 652$ cells (Jiang et al. 2011), where their applicability for bio-imaging was confirmed. The fluorescence emission was observed in that case around or inside the cells after their culturing with $\mathrm{ZnO}$ nanosheets.

\section{Conclusion}

The attractiveness of 6-TG as a drug in a combination with the possibility to use it as QDs surface modifier prompted us to demonstrate for the first time 6-TG-functionalized $\mathrm{CdSeS} /$ $\mathrm{ZnS}$ quantum dots as a promising tool for theranostics. The big advantage of the proposed approach is the double role of QDs, which allows for treating them as nanocarriers of a drug and tracking of distribution of obtained nanoconjugates by means of fluorescence imaging. We believe that the utilized conjugation chemistry (via direct interaction of sulfur-containing moiety with $\mathrm{ZnS}$ shell) contributes to the suppression of adverse drug properties resulting from the presence of thione group in the 6-TG structure.

Based on cytotoxicity measurements, a relationship between the concentration of QDs and cells viability was
Fig.7 QD-6-TG distribution monitoring

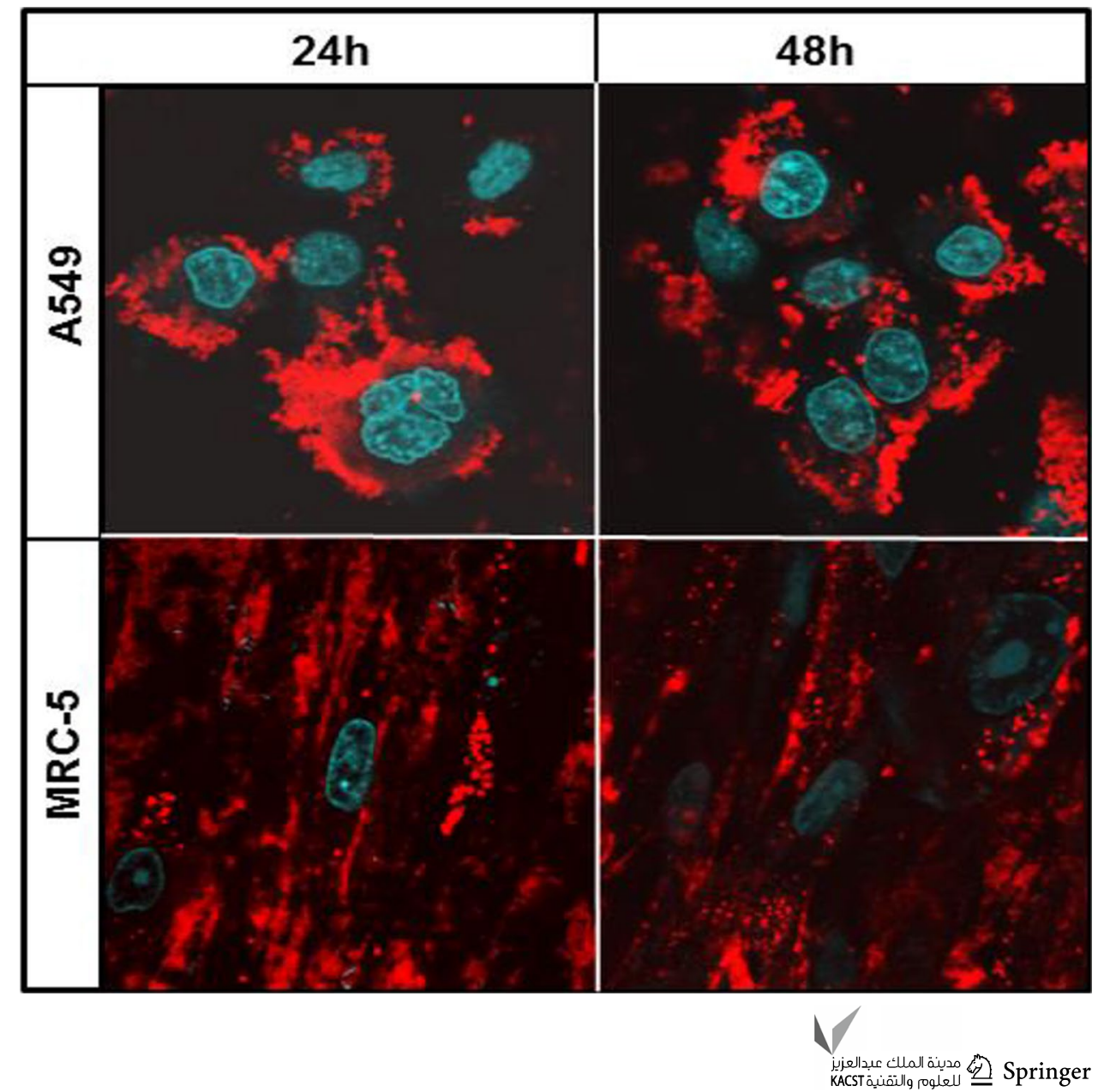


found. It was concluded that the increasing concentration of modified QDs as well as the longer incubation time caused reducing of cells viability. Moreover, it was found that drugmodified nanoparticles were the most toxic for lymphoblastic leukemia cells (K562); while in the case of normal lung cells (MRC-5), their cytotoxicity was the smallest. The fact that 6-TG surface-modified QDs are more toxic than the drug itself or QDs modified with mercaptoacetic acid has to be underlined as a main conclusion of this work.

Acknowledgements This work was accomplished with a frame of project SONATA, which is financed by the National Science Centre, contract No. UMO-2013/09/D/ST5/03832.

\section{Compliance with ethical standards}

Conflict of interest The authors declare that they have no conflict of interest.

Open Access This article is distributed under the terms of the Creative Commons Attribution 4.0 International License (http://creativeco mmons.org/licenses/by/4.0/), which permits unrestricted use, distribution, and reproduction in any medium, provided you give appropriate credit to the original author(s) and the source, provide a link to the Creative Commons license, and indicate if changes were made.

\section{References}

Abbasi E, Kafshdooz T, Bakhtiary M, Nikzamir N, Nikzamir N, Nikzamir M, Mohammadian M, Akbarzadeh A (2015) Biomedical and biological applications of quantum dots. Artif Cells Nanomed Biotechnol. https://doi.org/10.3109/21691401.2014.998826

Abdul Ghani SF, Wright M, Paramo JG, Bottrill M, Green M, Long N, Thanou M (2014) Three bisphosphonate ligands improve the water solubility of quantum dots. Faraday Discuss 175:153-169. https://doi.org/10.1039/c4fd00151f

Aghevlian S, Yousefi R, Faghihi R, Abbaspour A, Niazi A, Jaberipour M, Hosseini A (2013) The improvement of anti-proliferation activity against breast cancer cell line of thioguanine by gold nanoparticles. Med Chem Res 22:303-311. https://doi.org/10.1007/ s00044-012-0030-1

Antonello A, Guglielmi M, Bello V, Nobile Mattei GA, Martucci A (2011) Synthesis and tailoring of CdSe core@shell heterostructures for optical applications. In: Proceedings, integrated optics: devices, materials, and technologies XV, volume 7941; 794107. https://doi.org/10.1117/12.873678

Azzazy HME, Mansour MMH, Kazmierczak SC (2007) From diagnostics to therapy: prospects of quantum dots. Clin Biochem 40:917-927. https://doi.org/10.1016/j.clinbiochem.2007.05.018

Bera D, Qian L, Tseng T-K, Holloway PH (2010) Quantum dots and their multimodal applications: a review. Materials (Basel) 3:22602345. https://doi.org/10.3390/ma3042260

Biju V, Itoh T, Ishikawa M (2010) Delivering quantum dots to cells: bioconjugated quantum dots for targeted and nonspecific extracellular and intracellular imaging. Chem Soc Rev 39:3031. https:// doi.org/10.1039/b926512k

Bruchez M Jr (1998) Semiconductor nanocrystals as fluorescent biological labels. Science (80-) 281:2013-2016
Chalmers AH, Burdorf T, Murray AW (1972) Immunosuppression by 9-alkyl-6-thiopurines. Biochem Pharmacol 21:2662-2664. https ://doi.org/10.1016/0006-2952(72)90238-9

Deka S, Quarta A, Lupo MG, Falqui A, Boninelli S, Giannini C, Morello G, De Giorgi M, Lanzani G, Spinella C, Cingolani R, Pellegrino T, Manna L (2009) CdSe/CdS/ZnS double shell nanorods with high photoluminescence efficiency and their exploitation as biolabeling probes. J Am Chem Soc 131:2948-2958. https://doi. org/10.1021/ja808369e

Gao F (2011) Effects of quantum confinement and shape on band gap of core/shell quantum dots and nanowires. Appl Phys Lett 98:193105. https://doi.org/10.1063/1.3590253

Gutiérrez-Lazos CD, Ortega-López M, Rosendo E, Ortega-Avilés M, Sánchez-Reséndiz V, Hernández-Hernández A, MeléndezLira M, Matsumoto-Kuwabara Y, Espinoza-Rivas AM, PérezGuzmán MA (2012) Synthesis of CdTe quantum dots in aqueous solution and their optical and structural characterization. Sci Adv Mater 4:604-608. https://doi.org/10.1166/sam.2012.1326

ISO 10993-1:2018(en) Biological evaluation of medical devicesPart 1: evaluation and testing within a risk management process (n.d.). https://www.iso.org/obp/ui/\#iso:std:iso:10993:-1:ed5:v2:en. Accessed 17 Jan 2019)

Jiang H, Wang H, Wang X (2011) Facile and mild preparation of fluorescent $\mathrm{ZnO}$ nanosheets and their bioimaging applications. Appl Surf Sci 257:6991-6995. https://doi.org/10.1016/J.APSUS C.2011.03.053

Jin Z, Hildebrandt N (2012) Semiconductor quantum dots for in vitro diagnostics and cellular imaging. Trends Biotechnol 30:394403. https://doi.org/10.1016/j.tibtech.2012.04.005

Kalinowska D, Grabowska-Jadach I, Drozd M, Pietrzak M (2018) Comparative studies of biological activity of cadmium-based quantum dots with different surface modifications. Appl Nanosci 8:309-321. https://doi.org/10.1007/s13204-018-0787-8

Karabanovas V, Zitkus Z, Kuciauskas D, Rotomskis R, Valius M (2014) Surface properties of quantum dots define their cellular endocytic routes, mitogenic stimulation and suppression of cell migration., J Biomed Nanotechnol 10:775-786. http://www. ncbi.nlm.nih.gov/pubmed/24734530. Accessed 18 Jan 2019

Li J, Pu K (2019) Development of organic semiconducting materials for deep-tissue optical imaging, phototherapy and photoactivation. Chem Soc Rev 48:38-71. https://doi.org/10.1039/C8CS0 $0001 \mathrm{H}$

Li H, Chong X, Chen Y, Yang L, Luo L, Zhao B, Tian Y (2016) Detection of 6-thioguanine by surface-enhanced Raman scattering spectroscopy using silver nanoparticles-coated silicon wafer colloids surfaces a physicochem. Eng Asp 493:52-58. https:// doi.org/10.1016/J.COLSURFA.2016.01.032

Mottaghitalab F, Farokhi M, Fatahi Y, Atyabi F, Dinarvand R (2019) New insights into designing hybrid nanoparticles for lung cancer: diagnosis and treatment. J Control Release 295:250-267. https://doi.org/10.1016/j.jconrel.2019.01.009

Nanjwade BK, Singh J, Parikh KA, Manvi FV (2010) Preparation and evaluation of carboplatin biodegradable polymeric nanoparticles. Int J Pharm 385:176-180. https://doi.org/10.1016/j. ijpharm.2009.10.030

Nehate C, Jain S, Saneja A, Khare V, Alam N, Dubey R, Gupta $P$ (2014) Paclitaxel formulations: challenges and novel delivery options. Curr Drug Deliv 11:666-686. https://doi. org/10.2174/1567201811666140609154949

Podsiadlo P, Sinani VA, Bahng JH, Kam NW, Lee J, Kotov NA (2008) Gold nanoparticles enhance the anti-leukemia action of a 6-mercaptopurine chemotherapeutic agent. Langmuir 24:56857. https://doi.org/10.1021/la702782k

Selvaraj V, Grace AN, Alagar M, Hamerton I (2010) Antimicrobial and anticancer efficacy of antineoplastic agent capped gold 
nanoparticles. J Biomed Nanotechnol 6:129-37. http://www. ncbi.nlm.nih.gov/pubmed/20738066. Accessed 23 Jan 2019

Singla AK, Garg A, Aggarwal D (2002) Paclitaxel and its formulations. Int J Pharm 235:179-192. https://doi.org/10.1016/S0378 -5173(01)00986-3

Sleightholm L, Zambre A, Chanda N, Afrasiabi Z, Katti K, Kannan $R$ (2011) New nanomedicine approaches using gold-thioguanine nanoconjugates as metallo-ligands. Inorganica Chim Acta 372:333-339. https://doi.org/10.1016/j.ica.2011.01.065

Smith A, Duan H, Mohs A, Nie S (2008) Bioconjugated quantum dots for in vivo molecular and cellular imaging. Adv Drug Deliv Rev 60:1226-1240. https://doi.org/10.1016/j.addr.2008.03.015

Tamang S, Beaune G, Texier I, Reiss P (2011) Aqueous phase transfer of InP/ZnS nanocrystals conserving fluorescence and high colloidal stability. ACS Nano 5:9392-9402. https://doi.org/10.1021/ $\mathrm{nn} 203598 \mathrm{c}$

Wang R, Yue L, Yu Y, Zou X, Song D, Liu K, Liu Y, Su H (2016) Gold nanoparticles modify the photophysical and photochemical properties of 6-thioguanine: preventing DNA oxidative damage. J Phys Chem C 120:14410-14415. https://doi.org/10.1021/acs. jpcc.6b04277

Xia Y, Xu T, Zhao M, Hua L, Chen Y, Wang C, Tang Y, Zhu B (2018) Delivery of doxorubicin for human cervical carcinoma targeting therapy by folic acid-modified selenium nanoparticles. Int J Mol Sci 19:3582. https://doi.org/10.3390/ijms19113582
Xiao Y, Shi K, Qu Y, Chu B, Qian Z (2019) Engineering nanoparticles for targeted delivery of nucleic acid therapeutics in tumor. Mol Ther - Methods Clin Dev 12:1-18. https://doi.org/10.1016/j. omtm.2018.09.002

Yeon Woo J, Kumar Tripathy S, Kim K, Han C-S (2012) Thermal and structural dependence of the band gap of quantum dots measured by a transparent film heater. Appl Phys Lett 100:063105. https:// doi.org/10.1063/1.3682515

Zhang Y, Zhu X, Smith J, Haygood MT, Gao R (2011) Direct observation and quantitative characterization of singlet oxygen in aqueous solution upon UVA excitation of 6-thioguanines. J Phys Chem B 115:1889-1894. https://doi.org/10.1021/jp109590t

Zou X, Zhao H, Yu Y, Su H (2013) Formation of guanine-6-sulfonate from 6-thioguanine and singlet oxygen: a combined theoretical and experimental study. J Am Chem Soc 135:4509-4515. https:// doi.org/10.1021/ja400483j

Zrazhevskiy P, Sena M, Gao X (2010) Designing multifunctional quantum dots for bioimaging, detection, and drug delivery. Chem Soc Rev 39:4326. https://doi.org/10.1039/b915139g

Publisher's Note Springer Nature remains neutral with regard to jurisdictional claims in published maps and institutional affiliations. 